\title{
C.I. Lewis, E.J. Nelson, and the Modern Origins of Connexive Logic
}

Edwin Mares * and Francesco Paoli ${ }^{\dagger}$

Received: 9 December 2018 / Accepted: 11 February 2019

Abstract: Modern logic owes an important debt to C.I. Lewis and his students. In addition to Lewis's five modal logics, they are responsible for the creation (or discovery) of the logic of analytic implication and connexive logic. In this paper, we examine E.J. Nelson's connexive logic as an attempt to formalise the notion of entailment while avoiding the paradoxes of strict implication. We also look briefly at the reception of Nelson's logic and at Lewis's reply to it.

Keywords: Non-classical logic; C. I. Lewis; E. J. Nelson; relevant logic; paraconsistent logic; modal logic.

\section{Introduction}

In this paper, we explore the relationship between the formulation of a connexive logic in the early work of E.J. Nelson (as illustrated in Nelson 1929, 1930, 1933, 1936a) and C.I. Lewis's construction and understanding of his logic of strict implication. Before we begin to explore Nelson and Lewis's logical

\footnotetext{
${ }^{*}$ Victoria University of Wellington

Corresponding author. Philosophy Programme, P.O. Box 600 Wellington 6140, New Zealand

凶 edwin.mares@vuw.ac.nz

${ }^{\dagger}$ University of Cagliari

凶paoli@unica.it

(c) The Authors. Journal compilation () The Editorial Board, Organon F.
} 
views, here is some historical background. In the late 1920s and throughout the 1930s, a small school of logic students formed around C.I. Lewis in the Harvard Philosophy Department. The best known members of this school were William Parry, E.J. Nelson, and Daniel Bronstein, all of whom wrote theses on the nature of implication or entailment. Nelson (1900-1988), who is the subject of this essay, was from Washington State. Before coming to Harvard, he earned a bachelor's and master's degrees at the University of Washington. He studied at Harvard from 1925 to 1929, where he earned his PhD. He then was granted a travelling scholarship by Harvard, which he took at Göttingen and the Sorbonne, before taking up a teaching position at the University of Washington. He taught there until 1952, when he moved to Ohio State University, where he taught until his retirement in $1968 .{ }^{1}$

Connexive logic is characterised as having some or all of the following principles. Aristotle's theses state that no proposition entails its own negation:

$$
\begin{aligned}
& \neg(A \rightarrow \neg A) \\
& \neg(\neg A \rightarrow A)
\end{aligned}
$$

And Boethius's theses state that no proposition entails both a proposition and its negation:

$$
\begin{aligned}
& (\mathrm{A} \rightarrow B) \rightarrow \neg(A \rightarrow \neg B) \\
& (\mathrm{A} \rightarrow \neg B) \rightarrow \neg(A \rightarrow B)
\end{aligned}
$$

As the names of these theses suggest, connexive logic has its roots in ancient philosophy. These theses were adopted and logics contained them were developed by mediaeval logicians such as Abelard. ${ }^{2}$

In his $\mathrm{PhD}$ thesis and elsewhere, Nelson motivates his approach to logic by claiming that it avoids the paradoxes of strict implication, namely: that every proposition entails every logical truth and that a contradiction entails every proposition. Lewis famously gives an argument for the acceptance of the latter paradox in Symbolic Logic. Perhaps less known is his argument for the same paradox in his 1918 book, A Survey of Symbolic Logic. It is the argument in the Survey to which Nelson reacts, and so we examine it in section 2 below.

\footnotetext{
${ }^{1}$ The information here was obtained from a biography of Nelson found at https://www.pdcnet.org/

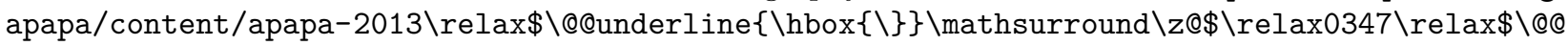
underline $\{\backslash$ hbox $\{\backslash\}\} \backslash \operatorname{mathsurround} \backslash z @ \$ \backslash \operatorname{relax} 0348 \backslash \operatorname{relax} \$ \backslash @ @$ underline $\{\backslash$ hbox $\{\backslash\}\} \backslash \operatorname{mathsurround} \backslash$ z@\$\relax547?file\{\\}type=pdf.

${ }^{2}$ For general histories of connexive logic, see (McCall 2012) and (Wansing 2016).
} 
Nelson's logic, as we shall argue, is not just a reaction against the adoption of the paradoxes of strict implication by his supervisor, C.I. Lewis, but also an attempt to recast Lewis's project of formulating a logic of entailment. For Nelson, ' $A \rightarrow B$ ' expresses that $B$ is deducible from $A$ in some intuitive sense of 'deducibility'. If we take the arrow to mean strict implication, this is true for Lewis as well.

Nelson follows Lewis by beginning with a notion of consistency and defining an entailment connective in terms of it and negation. Nelson's consistency connective, however, is more intensional than Lewis's, and its use results in a very different logic of entailment. Overall, Nelson's treatment of the connectives is far more intensional than Lewis's. His treatment of conjunction, in particular, is intensional. He rejects the thesis of simplification, $(A \wedge B) \rightarrow A$, even though he admits that if $A \wedge B$ is true then $A$ must be true. His approach to entailment, and logic more generally, is not truth-conditional in any ordinary sense. As we shall see, the notions of intension and meaning are of central importance to Nelson's approach to logic and motivate the system that he accepts.

Although Lewis rejects Nelson's logic, he does come to accept a philosophy of logic that is very intensional in the manner of Nelson's philosophical views. They come to use very similar language to describe what logic is about.

In this paper we examine the system that Nelson puts forward in his $\mathrm{PhD}$ thesis and in (Nelson 1930). ${ }^{3}$ We think that examining his actual system sheds a lot of light on his thinking behind his advocacy of connexive logic.

\section{The early argument for explosion ${ }^{4}$}

In chapter V, section V of the Survey of Symbolic Logic, Lewis states that two paradoxes of strict implication in fact are theorems of his system (Lewis 1918, 335):

$$
3.52 \sim p-3(p-3 q)
$$

\footnotetext{
${ }^{3}$ The system in the thesis seems to have a rule of adjunction (which is strangely formulated) but this does not appear in (Nelson 1930).

${ }^{4}$ We refer to this argument as "early" because it is presented 14 years earlier than his more famous argument of (Lewis 1951)
}

Organon F 26 (3) 2019: 405-426 


\section{$3.55 \sim \neg p \neg(q \neg p)$.}

In (Lewis 1918), " " means "it is impossible that". So, 3.52, says that if $p$ is impossible, then it entails everything. " $\sim \neg p$ " means " $p$ is necessary", so 3.55 means that if $p$ is necessary, then it is entailed by every proposition.

The argument that Lewis gives in section $\mathrm{V}$, however, does not prove either 3.52 or 3.55. Rather, it justifies the thesis form of explosion (the proposition that a contradiction entails everything, as opposed to a rule form according to which contradictory theorems entail that everything is a theorem). The argument begins with the following three principles.

$$
\begin{aligned}
& \text { P1 }(p \wedge q) \neg q \\
& \text { P2 }((p \wedge q) \neg r) \neg((p \wedge \neg r) \neg \neg q) \\
& \text { P3 }(((p \wedge q) \neg r) \wedge(r \neg s)) \neg((p \wedge q) \neg s)
\end{aligned}
$$

$\mathrm{P} 1$ is just the principle of simplification. We discuss this at some length below. P2 is the principle of antilogism. As we shall see presently, it is a logical principle that Lewis and his school took to be a central law of logical reasoning. P3 is just an instance of Lewis's transitivity axiom. Lewis also appeals to uniform substitution, the replacement of equivalents, and a rule form of transitivity of -3 .

Here is Lewis's argument as presented in (Lewis 1918, 336):

1. $(((p \wedge q)-3 q) \wedge(q-3 r))-3((p \wedge q)-3 r) \quad \mathrm{P} 3$

2. $((p \wedge q)-3 r)-3((q \wedge \neg r) \neg \neg p) \quad \mathrm{P} 2$

3. $(((p \wedge q) \neg q) \wedge(q \neg r)) \neg((q \wedge \neg r) \neg \neg p) \quad 1,2$, transitivity rule

By substituting $q$ for $r$ we obtain:

$$
(((p \wedge q) \neg q) \wedge(q \neg q)) \neg((q \wedge \neg q) \neg \neg p)
$$

By P1, $(p \wedge q) \neg q$ is a theorem. And Lewis assumes that $q \neg q$ is a theorem. Hence, by the rule of adjunction, $(((p \wedge q)-3 q) \wedge(q-3 q))$ is a theorem, and so, by modus ponens,

$$
(q \wedge \neg q) \neg \neg p
$$

is provable. As Lewis says " $p$ itself may be negative" and so "this impossible proposition implies anything" (ibid.). This last step appeals to the equivalence 
of a proposition and its double negation and the substitutivity of propositions and their logical equivalents. ${ }^{5}$

In his early work (Nelson 1929; 1930), Nelson rejects P1 and modifies P3 in his logic. He discusses P2 at length, both in his thesis and in (Nelson 1933), but he retains it. As we have said, the principle of antilogism is taken by Lewis to be a central principle of deductive inference. The historical reasons for this have to do with the work of Christine Ladd-Franklin (1983), who reconstructed traditional syllogisms as "inconsistent triads" of propositions. For example, Barbara is of course

Every $A$ is $B$
Every $B$ is $C$
$\therefore$ Every $A$ is $C$

Construed as an inconsistent triad it becomes

$$
\begin{aligned}
& \text { Every } A \text { is } B \\
& \text { Every } B \text { is } C \\
& \text { Some } A \text { is not } C
\end{aligned}
$$

Any two of these propositions entails the negation of the third. Hence, the valid syllogisms that can be generated are:

- Every $A$ is $B$, every $B$ is $C \therefore$ every $A$ is $C$;

- Every $A$ is $B$, some $A$ is not $C \therefore$ some $B$ is not $C$;

- Every $B$ is $C$, some $A$ is not $C \therefore$ some $A$ is not $B$.

Lewis cites Ladd-Franklin's reasoning about syllogisms with approval both in the Survey (Lewis 1918, 108-110) and in Symbolic Logic (Lewis and Langford 1951, 60-61). Nelson seems to have followed him in thus treating the antilogism as a key deductive principle.

${ }^{5}$ Given these assumptions, the argument can be made much simpler:

$$
\begin{array}{lll}
\text { 1. } & (q \wedge \neg p)-3 q & \text { P1, US } \\
\text { 2. } & (q \wedge \neg q)-\neg \neg \neg p & 1, \text { P2, MP } \\
\text { 3. } & (q \wedge \neg q)-\neg p & 2, \text { DN, Eq }
\end{array}
$$


Antilogism has a mixed reception in Lewis's school. Nelson considers modifying it in (Nelson 1933). William Parry - a student of Lewis's in the early 1930s - criticises antilogism because it clashes with the key idea behind his logic of analytic implication. On Parry's view, no implication $A \rightarrow B$ is a theorem unless all the propositional variables in $B$ occur in $A$. But, if we accept antilogism, we may derive implications that disrespect Parry's principle from implications that respect it. For example, $(A \wedge B) \rightarrow C$ may be such that $\operatorname{Var}(C) \subseteq \operatorname{Var}(A) \cup \operatorname{Var}(B)$, but this is no guarantee that $\operatorname{Var}(\neg B) \subseteq \operatorname{Var}(A) \cup \operatorname{Var}(\neg C)$ in $(A \wedge \neg C) \rightarrow \neg B$.

\section{Consistency and entailment}

Nelson's approach to logic is extremely intensional. Nelson not only interprets the entailment connective as an intensional operator, but he also interprets conjunction intensionally:

I do not take $p \wedge q$ to mean " $p$ is true and $q$ is true", but simply " $p$ and $q$ ", which is a unit or whole, not simply an aggregate, and expresses the joint force of $p$ and $q$. (Nelson 1930, 444)

How to understand the notion of the "joint force" of $p$ and $q$ is unclear. Nelson postulates very few axioms for conjunction in his logic (see $\S 4$ ), so we know very little about its nature.

Nelson uses his intensional conjunction to reject the thesis of simplification $((p \wedge q) \rightarrow p):$

Naturally, in view of the fact that a conjunction must function as a unity, it cannot be asserted that the conjunction of $p$ and $q$ entails $p$, for $q$ may be totally irrelevant to and independent of $p$, in which case $p$ and $q$ do not entail $p$, but it is only $p$ that entails $p$. I can see no reason for saying that $p$ and $q$ entail $p$, when $p$ alone does and $q$ is irrelevant, and hence does not function as a premise in the entailing. (Nelson 1930, 447)

In this passage, Nelson alludes to some strong form of real use criterion for premises in an inference. The proposition $p \wedge q$ can only entail $r$ when both $p$ 
and $q$ are really used in the derivation. As we shall see in section 4 below, it would seem that Nelson's notion of real use is very strict indeed.

It is interesting to compare Nelson's conjunction with the intensional conjunction of relevant and linear logic. The intensional conjunction of relevant and linear logic is called "fusion". Here we represent it by $\otimes$. Like Nelson's conjunction, fusion does not satisfy simplification, that is, $(p \otimes q) \rightarrow p$ is not valid in relevant or linear logics. Nelson's connective, however, satisfies the principle of idempotence:

$$
p \rightarrow(p \wedge p)
$$

In strong relevant logics like the the logic $\mathrm{R}$ of relevant implication or $\mathrm{E}$ of relevant entailment, fusion is idempotent. But it is not in linear logic or in weaker relevant logics. Idempotence $(p \rightarrow(p \otimes p))$ in these logics is equivalent to contraction:

$$
(p \rightarrow(p \rightarrow q)) \rightarrow(p \rightarrow q)
$$

Moreover, adding contraction to these logics, by Curry's paradox, trivialises naïve theories of truth, sets, or properties.

Nelson observes that the idempotency of intensional conjunction, together with antilogism, yields undesirable outcomes. Tweaking Nelson's example slightly, "Rome is in Italy" entails "Rome is in Europe". However, if "Rome is in Italy" is equivalent to "Rome is in Italy and Rome is in Italy", then the latter also entails "Rome is in Europe", whence antilogism gives that "Rome is in Italy and Rome is not in Europe" entails "Rome is not in Italy". This, however, is absurd, because our conclusion does not follow from the "joint force" of the conjuncts. Nelson, however, does not go on to question idempotency of his intensional conjunction. Rather, he seems to suggest that "Rome is in Italy and Rome is in Italy" is actually a simple sentence, and not a conjunction of two simple sentences:

If at any place in a symbolic statement, an additional mark does not add to the logical significance, that mark should be dropped out. [...] We might formulate this as a principle of significance: $p \wedge p$ must be given no significance, either in operations or in inferences, beyond that possessed by $p$. (Nelson 1933, 281)

Given his view that ' $p \wedge p$ ' says no more than ' $p$ ', conjunction must be idempotent.

Organon F 26 (3) 2019: 405-426 
In the mid-1930s, however, Nelson's opposition to simplification begins to waver. He says that simplification is more intuitive than addition $(p \rightarrow$ $(p \vee q))$, and suggests that addition may be abandoned and that either uniform substitution be restricted or that antilogism be restricted with a consistency proviso about the sentences at issue (Nelson 1933, 279). ${ }^{6}$ We think that he may have been influenced in this by Parry. Parry's analytic implication severely restricts addition, in an attempt to avoid the paradoxes of strict implication. Parry's thesis was defended in 1931, and so its ideas and results may not have been known to Nelson until well after he wrote his own dissertation and (Nelson 1930).

In this paper, we concentrate on Nelson's entailment and consistency operators as they are developed in (Nelson 1929; 1930). These two closely linked connectives are the central concern of most of Nelson's writings about connexive logic. In addition, Nelson's critique of Lewis concentrates on the latter's treatment of these two connectives.

First, let us look at Lewis. In the Survey, Lewis defines the consistency connective (o) as follows:

$$
A \circ B=_{d f} \neg \sim(A \wedge B)
$$

But we interpret this definition in terms of possibility and standard (extensional) negation:

$$
A \circ B={ }_{d f} \diamond(A \wedge B)
$$

In the Survey, Lewis understands possibility in syntactic terms. He defines a "possible situation" as a consistent set of propositions. An "impossible situation" is an inconsistent set of propositions (Lewis 1918, 333). Then, $A \circ B$ is true if and only if there is some possible situation that contains both $A$ and $B$.

Strict implication is defined in terms of consistency and extensional negation:

$$
A \neg B={ }_{d f} \neg(A \circ \neg B)
$$

Lewis interprets strict implication in terms of situations by saying that $p-3 q$ is true if and only if any situation in which $p$ is true and $q$ is false is impossible (ibid.). Lewis's treatment of possibility, consistency, and strict implication is

\footnotetext{
${ }^{6}$ In a letter to the editors of Mind in 1936, Nelson says "I still believe, however, that $(p \wedge \neg p) \rightarrow q$ is unacceptable, but that it can be avoided by means other than the rejection of $(p \wedge q) \rightarrow p$ " (Nelson 1936b).
} 
not semantic in the modern sense. Rather these notions are understood in terms of sets of propositions from which we can or cannot derive contradictions. The logic used to determine what sets are consistent is the logic of strict implication itself (see Lewis 1923a). Whether the sort of circularity involved here is vicious is not our current concern. Rather, we just wish to indicate the ways in which Nelson's view is different from Lewis's.

Nelson's approach to consistency and inconsistency is much less formal than Lewis's. We think that Nelson's notion of inconsistency is more accurately represented as a relation of conflict between meanings, rather than consistency in any standard sense. ${ }^{7}$ For the most part, Nelson thinks that we need to examine the meanings of sentences to determine whether they are in conflict with one another. This is an informal procedure, in the sense that it cannot be fully represented in a formal system. Nelson, however, does give us some structural constraints on this notion of conflict, and these constraints are represented formally. Here are three of the most important constraints:

1. No proposition conflicts with itself.

2. Every proposition conflicts with its own negation.

3. No proposition entails any proposition with which it conflicts.

Nelson uses Lewis's notation (o) for consistency (i.e. failure of conflict). He defines conflict (|) as

$$
A \mid B=_{d f} \neg(A \circ B)
$$

and he defines his entailment connective in terms of conflict. We use ' $\rightarrow$ ' for Nelson's entailment. He used ' $E$ ', but we find that rather ugly. Here is the definition of entailment:

$$
A \rightarrow B=_{d f} \quad A \mid \neg B
$$

The second constraint is an axiom of Nelson's logic:

$$
p \rightarrow p
$$

\footnotetext{
${ }^{7}$ Nelson's glosses on consistency and conflict are rather enigmatic. For example (Nelson 1936a, 507): "even if $p$, possibly $\neg q$ " is a reading of ' $p \circ \neg q$ '. The use of 'even if' and the obvious scope problems concerning 'possibly' in this formulation obscure its meaning. We think the notion of conflict and lack of conflict make more sense of $\mid$ and $\circ$.
}

Organon F 26 (3) 2019: 405-426 
i.e. $p \mid \neg p$. The third constraint is also an axiom:

$$
(p \rightarrow q) \rightarrow(p \circ q)
$$

By uniform substitution and modus ponens, we then can derive the first constraint:

$$
p \circ p
$$

or, in terms of conflict (using double negation introduction):

$$
\neg(p \mid p)
$$

Thus no proposition conflicts with itself. Even contradictions fail to conflict with themselves. Nelson's notion of conflict is not truth conditional, unlike Lewis's notion of consistency. On Lewis's view, as we have seen, two propositions are consistent if and only if they can be in the same possible situation. A contradiction cannot be in a possible situation. The notion of conflict has to do with oppositions between the contents of propositions.

Graham Priest has suggested that negation in connexive logic be interpreted as "cancellation" negation (Priest 1999). On this view, contradictions are taken to have no content. We do not think that Nelson interpreted his negation in this way (although this is not to say that this might be a better way to interpret his negation). Contradictions, like all other statements, express propositions, and propositions are taken to have positive contents, in some sort of Fregean sense. Nelson does not consider the amount of content a statement has. He has a purely qualitative understanding of content. ${ }^{8}$

The difference in approach to contradictions/conflicts is part of a basic difference in philosophy of logics between Nelson and Lewis. In the 1920s, Lewis adopts a pragmatic approach to analyticity, apriority, and logical truth (Lewis 1923b; 1930; 1929). Part of this pragmatic conception is that analyticity and logical truth are conventional. In the early 1920s, Lewis develops his earlier notion of situations into what he calls systems. Systems are logically closed sets of states of affairs (Lewis 1923a). ${ }^{9}$ On the pragmatic view, which sets of states of affairs count as systems is to a large extent a matter of convention.

\footnotetext{
${ }^{8}$ Priest's reading of connexive logic is rather post-Carnap/Bar Hillel. It treats the semantics of sentences in terms of how much information they convey. Priest's interpretation of connexive logic may be more fruitful, but our purpose here is purely historical and we only wish to examine what Lewis and Nelson are doing.

${ }^{9}$ Lewis uses the terminology "facts" for what we call "states of affairs". The reason we do not adopt his terminology is that "fact" now generally refers to entities that are true whereas states of affairs need not be true.
} 
For Nelson, on the other hand, which intensional relations (such as conflict) hold between propositions is evident to one in an act of immediate intuition, which is independent of "any acquaintance with particulars" (Nelson 1929, 3 ). Thus, whereas Lewis has a pragmatic and conventionalist approach to the philosophy of logic, Nelson takes a hard realist-platonist approach.

Before we move on to our presentation of Nelson's logic, we indulge ourselves in a bit of speculation. In an appendix to Symbolic Logic, Lewis formulates four logics in addition to his favourite system of strict implication, which at that time is S2. He wishes to avoid committing himself to the following strong form of transitivity of $\rightarrow$ (Lewis and Langford 1951, 496):

$$
(p-3 q)-3((q-3 r)-3(p-3 r))
$$

When writing the first edition of Symbolic Logic in the early 1930s, Lewis has no proof that this form of transitivity cannot be derived in S2. He knows that it can be derived in S3. He also can prove that it does not follow in S1, which results from dropping the axiom

$$
(*) \diamond(p \wedge q)-\diamond \diamond p
$$

from S2. Lewis suggests that S1 be adopted if it is found that S2 contains strong transitivity. The axiom $*$, however, is rather intuitive and dropping it has seemed to most logicians a bad price to pay to avoid strong transitivity. The intuitiveness of $*$ rests on the reading of $\diamond$ as possibility or as consistency (which is the same thing for Lewis). If, on the other hand, we read $\diamond(p \wedge q)$ as saying that $p$ does not conflict with $q$ we may not think that $*$ is valid. For the content of $p$ and $q$ may not conflict, but $p$ may conflict with itself. It may be, then, that Nelson had some influence on Lewis in the latter's formulation of S1. Whether this is really the case, we have no strong evidence.

\section{Nelson's logic}

In this section, we present Nelson's connexive logic, which we call 'NL'.

We change Nelson's notation slightly, using $\wedge$ instead of mere concatenation, $\rightarrow$ instead of $E$, and $\neg$ instead of - . Nelson's primitive connectives are 
$\neg, \circ$, and $\wedge$. Inconsistency is defined, quite reasonably, using consistency and negation:

$$
A \mid B=_{d f} \neg(A \circ B)
$$

and entailment is defined in terms of inconsistency:

$$
A \rightarrow B={ }_{d f} \quad A \mid \neg B
$$

Intensional logical equivalence or the identity of meaning, $=$, is defined as usual:

$$
A=B=_{d f}(A \rightarrow B) \wedge(B \rightarrow A)
$$

Nelson also defines an "intensional sum" (an intensional disjunction), $\vee$ :

$$
A \bigvee B=_{d f} \neg A \mid \neg B
$$

We do not discuss intensional sum in what follows, and it does not appear in any of his axioms. In the axiomatisation of his logic Nelson does use an unusual abbrevation, $A \neq B \neq C$. It is defined as follows:

$$
A \neq B \neq C=_{d f}(A \neq B) \wedge(B \neq C) \wedge(A \neq C) .
$$

Nelson set out his system on pages 138-141 of his thesis.

\section{Axioms of NL}

$1.1 p \rightarrow p$

$1.2(p \mid q) \rightarrow(q \mid p)$

$1.3 p \rightarrow \neg \neg p$

$1.4(p \rightarrow q) \rightarrow(p \circ q)$

$1.5(p \neq q \neq r) \rightarrow(((p \rightarrow q) \wedge(q \rightarrow r)) \rightarrow(p \rightarrow r))$

$1.6(p \wedge q)=(q \wedge p)$

$1.7((p \wedge q) \rightarrow r) \rightarrow((p \wedge \neg r) \rightarrow \neg q)$

We have a few comments to make on the axiom list before we go on to state the rules of NL.

Axiom 1.1, stated in terms of the conflict connective, is

$$
p \mid \neg p,
$$


which merely states that every proposition conflicts with its own negation. Axiom 1.2 states that conflicts are symmetrical. 1.3 is not justified in terms of the notion of conflict, but rather in terms of an intuition about negation. As we shall seen below, the converse of 1.3 is also provable and the meaning of any proposition according to this logic is the same as the meaning of its double negation.

Axiom 1.4 is the only contra-classical axiom in the logic. We discussed it in section 3 above. It says that if either of two propositions entails the other, then they do not conflict with one another. Together with 1.1, 1.4 entails that no proposition conflicts with itself.

Nelson prefixes his transitivity axiom (1.5) with $p \neq q \neq r$. If we eliminate the prefix, then we get theorems like

$$
((p \rightarrow p) \wedge(p \rightarrow q)) \rightarrow(p \rightarrow q),
$$

that are instances of the simplification scheme that Nelson rejects. Daniel Bronstein (another of Lewis's students) accuses Nelson of committing a fallacy here (Bronstein 1936, 167-168). Bronstein points out that just because unconstrained transitivity allows the derivation of some instances of simplification does not mean that it allows the derivation of all instances. In particular, it is not shown that unrestricted transitivity will allow the derivation of any paradoxes. Moreover, one might wonder, however, whether this thesis is as objectionable as other cases of simplification, since we can think of $p \rightarrow p$ being used (rather inefficiently) to derive $p \rightarrow q$ from itself by transitivity. That is, we do not have to think of the derivation of $p \rightarrow q$ from $(p \rightarrow p) \wedge(p \rightarrow q)$ merely as a dropping of the first conjunct of the premise.

The inclusion of the prefix in this axiom ${ }^{10}$ may indicate that Nelson has in mind a very strong form of real-use principle. The above instance of transitivity might be thought to be objectionable because $p \rightarrow p$ is not needed (although it may be used) to derive $p \rightarrow q$. This very strong version of the principle says that $\left(A_{1} \wedge \ldots \wedge A_{n}\right) \rightarrow B$ can be allowed as a theorem only if there is no proper subset $\{i, \ldots, j\}$ of $\{1, \ldots, n\}$ such that $\left(A_{i} \wedge \ldots \wedge A_{j}\right) \rightarrow B$ is also a theorem. This is a very strong requirement indeed, and we do not have enough evidence to attribute it to Nelson.

The antilogism axiom (1.7) seems even more natural if read in terms of

\footnotetext{
${ }^{10}$ Which he continues to accept in (Nelson 1933).
} 
conflict (modulo some fiddling with double negations):

$$
((p \wedge q) \mid r) \rightarrow((p \wedge r) \mid q)
$$

This is very close to Ladd-Franklin's formulation of her principle of inconsistent triads.

NL does not contain an axiom, such as, $(p \wedge(q \wedge r)) \rightarrow((p \wedge q) \wedge r)$, that states that conjunction is associative. His use of strings of conjunctions without parentheses makes it seem as though he thought of conjunction as associative, but it is difficult to say whether the omission of an associativity axiom was an oversight or is intentional.

\section{Rules}

The rules that Nelson states in his axiomatisation are the following:

$$
\frac{\vdash A \rightarrow B \quad \vdash A}{\vdash B} \quad \frac{\vdash A \vdash B}{\vdash A \wedge B}
$$

We abbreviate 'modus ponens' as 'MP' and 'adjunction' as 'Adj'.11

In his proofs of theorems, Nelson also makes free use of two substitution rules: a standard rule of uniform substitution for propositional variables ('US') and a rule of substitution for logical equivalents. This latter rule, for which we adopt the name 'Eq' from (Hughes and Cresswell 1968), is the following:

$$
\frac{\vdash A=B}{\vdash C=C^{\prime}}
$$

where $C^{\prime}$ results from replacing one or more occurrences of $A$ with $B$ in $C$.

$\mathrm{NL}$, as we think of it, then contains axioms 1.1-1.7, the rules MP, Adj, US, and Eq.

\footnotetext{
${ }^{11}$ Nelson's statement of Adj is somewhat convoluted: "If a proposition, or all the propositions of a logical product, are asserted, separately, or otherwise, but as such, then whatever is entailed by or is identical with that proposition, or that logical product, as the case may be, is categorically asserted" (Nelson 1929, 141). Clearly this statement includes Adj, but also a rule that says that whatever is entailed by a theorem is a theorem. This is included in the rule MP, so we do not need to state it independently.
} 
Here is a proof of the left to right half of the principle of double negation (DN) $(A \rightarrow \neg \neg A)$ (taken, in effect, from Nelson 1929, 159):

1. $\neg A \rightarrow \neg A$ 1.1, uniform substitution

2. $\neg A \mid \neg \neg A \quad 1$, def. of $\rightarrow$

3. $\neg \neg A \mid \neg A \quad 2,1.2, \mathrm{MP}$

4. $\neg \neg A \rightarrow A$ 3, def. of $\rightarrow$

Together with axiom 1.3 and Adj, we obtain

$$
\vdash p=\neg \neg p .
$$

We call this 'DN' and we use it extensively in our other proofs. For example, here is a proof of contraposition:
1. $(p \mid \neg q) \rightarrow(\neg q \mid p)$
1.2 , US
2. $(p \mid \neg q) \rightarrow(\neg q \mid \neg \neg p)$
1, DN, Eq
3. $(p \rightarrow q) \rightarrow(\neg q \rightarrow \neg p)$ 2, def. of $\rightarrow$, Eq

\section{Nelson's logic as a connexive logic}

A connexive logic is characterised as a system which accepts some or all of Aristotle's and Boethius's theses. Nelson's axiom 1.4 is

$$
(p \rightarrow q) \rightarrow(p \circ q)
$$

By the definition of conflict, DN, and Eq, we obtain

$$
(p \rightarrow q) \rightarrow \neg(p \mid q)
$$

and so, by DN, the definition of implication, and Eq we get

$$
(p \rightarrow q) \rightarrow \neg(p \rightarrow \neg q) .
$$

This is Boethius's first thesis. In fact, 1.4 is equivalent to Boethius's first thesis:

1. $(p \rightarrow q) \rightarrow \neg(p \rightarrow \neg q)$ Boethius

2. $(p \rightarrow q) \rightarrow \neg(p \mid q) \quad$ 1, DN, Eq, def. of $\} \rightarrow$

3. $(p \rightarrow q) \rightarrow(p \circ q) \quad 2$, def. of $\circ$

Organon F 26 (3) 2019: 405-426 
The second thesis can be derived from the first by contraposition, DN, and Eq:

$$
(p \rightarrow \neg q) \rightarrow \neg(p \rightarrow q)
$$

In (Nelson 1936a), he argues for Boethius's theses. He does so by reading $\rightarrow$ as 'if ... then'. Nelson objects to the reading of 'if ... then' as material implication. He claims that when one denies that 'if $p$, then $q$ ' she is not asserting $p$ and $\neg q$. When one denies 'if the ground is wet, then it is raining', one does not assert that the ground is wet. She may only be denying the connection between the ground's being wet and rain. Nelson asserts that the contradictory of 'if $p$, then $q$ ' is ' $p \circ \neg q$ ' (Nelson 1936a, 507). The contradictory of $p \circ \neg q$ is $\neg(p \circ \neg q)$, i.e., $p \rightarrow q$, hence the proper treatment of 'if .. then' in English (at least in some cases) is as $\rightarrow$.

Aristotle's first thesis follows straightforwardly from Boethius's first thesis:

1. $p \rightarrow p$

1.1

2. $(p \rightarrow p) \rightarrow \neg(p \rightarrow \neg p)$ Boethius's first thesis and US

3. $\neg(p \rightarrow \neg p)$ $1,2, \mathrm{MP}$

Aristotle's second thesis follows in the same way, but with the use of $\neg p \rightarrow \neg p$ as a substitution instance of 1.1 and substituting $\neg p$ for $p$ in Boethius's first thesis.

NL altered by the removal of the prefix $(p \neq q \neq r)$ from axiom 1.5 to produce a standard form of conjunctive syllogism $(((p \rightarrow q) \wedge(q \rightarrow r)) \rightarrow$ $(p \rightarrow r)$ ), yields a subsystem of Richard Routley and Hugh Montgomery's connexive logic Z1c (Routley and Montgomery 1968). Let us call this logic with the removal of the prefix from $1.5, \mathrm{NL}^{-}$. This logic is very closely related to weak relevant logics. If we define $A \circ B$ as $\neg(A \rightarrow \neg B)$ and $A \mid B$ as $A \rightarrow \neg B$, then all the axioms of $\mathrm{NL}^{-}$, except 1.4 , are provable in the relevant logic DK. Whether NL (or even $\mathrm{NL}^{-}$) should be considered a relevant logic is a technical issue that we set aside here.

\section{Contemporary connexive approaches}

Soon after Nelson finished his dissertation and published (Nelson 1930), some other philosophers adopted similar views. 
According to (Baylis 1931), it is true that $p$ implies $q$ iff $p$ is inconsistent with $\neg q$, but this is rather a consequence of the definition of implication rather than the definition itself. We have that $p$ implies $q$ (or else, is subsumed under $q$ ) in case the intensional meaning of $q$ is identical with a part of the intensional meaning of $p$.

(Wisdom 1934) criticises Lewis' definition of consistency, taking sides with Nelson also on the issue of paradoxes of strict implication.

Duncan-Jones claims to have arrived independently at an analysis of paradoxes similar to Nelson's:

The general point of view which I am adopting is much the same as that of Everett J. Nelson in "Intensional relations", Mind 1930. I had already worked out this paper in outline before I discovered the existence of Nelson's paper: otherwise the note would have been based directly on Nelson. I differ from Nelson on one or two points to be mentioned below. (Duncan-Jones 1935, 70)

Such points are actually not quite minor. Duncan-Jones expresses doubts about Nelson's intensional understanding of conjunction and about his ensuing rejection of conjunctive simplification. To rescue this principle, Duncan-Jones distinguishes between implying and invoking. We have that $p$ invokes $q$ if $p$ entails $q$ and $p$ is not a conjunction which would still entail $q$ if one of its conjuncts were dropped. The paradoxes of strict implication, thus, are fallacies of equivocation: conjunctive simplification holds for implication but not for invocation, while antilogism holds for the latter but not for the former.

\section{Lewis's reply to Nelson}

Lewis does not reply to Nelson by name. In fact, Lewis rarely publishes explicit replies to criticisms of either his logical or philosophical views. But we do find in his work, some discussion and a clear rejection of connexive logic.

Lewis briefly discusses Nelson's axiom 1.4 in Symbolic Logic (Lewis and Langford 1951, 157):

The principle $(p-3 q)-3(p \circ q)$, which might be expected to hold, does not, in fact, hold without exceptions. There are propositions which are not consistent with any other propositions; yet such 
propositions have implications. Hence if $p-3 q$ holds, it requires the further condition $p \circ p$ to assure that $p$ is consistent with $q$.

He also argues against Aristotle's thesis. He points out that, in his system, whenever $p \neg q$ is a theorem, $p \wedge \neg q$ entails its own negation.

In an appendix to the second edition of Symbolic Logic written in January 1959, he acknowledges that "on account of the paradoxes there are many who doubt that" S2 is the logic of entailment, that it captures the intuitive notion of deducibility. He points out that there are alternative logics that avoid the paradoxes but that these all fail to contain certain "indispensable rules of inference" (Lewis and Langford 1959, 511-512).

As we have said, Lewis does not mention Nelson by name, but he attacks Nelson's rejection of the simplification thesis:

If the moon is a planet and not made of green cheese, then the moon is a planet. But if two premises together imply a conclusion and the conclusion is false while one of the premises is true, then the other premise must be false. So if the moon is a planet and also is not a planet, then the moon is made of green cheese. We need not dally with a suppositious amendment: "Whatever in the premises is non-essential to the conclusion is no part of what implies the conclusion." This dictum would condemn the syllogism, since in every syllogism the premises contain information not contained in the conclusion. (Lewis and Langford 1959, 512) ${ }^{12}$

In this passage, Lewis is attributing to Nelson the requirement that whatever information is in the premises must appear in the conclusion. We doubt that Nelson in fact held this. But if he did, surely the only general principle of logic that he could accept is the thesis of identity, i.e., $p \rightarrow p$, and Lewis says that this is all that can be justified if we accept the "suppositious amendment" (ibid.). We interpret Nelson as holding a strong form of real use requirement, that all the premises need to be used to derive a conclusion, not that all the information in the premises survives in the conclusion.

At any rate, Lewis clearly rejects connexive logic because he thinks that simplification is an indispensable rule of inference, and that the corresponding

\footnotetext{
${ }^{12}$ Notice the use of antilogism in this passage. The argument is in fact a truncated version of Lewis's early independent argument discussed in section 2.
} 
thesis, $(p \wedge q) \rightarrow p$, is required by a logic that is meant to capture the intuitive notion of deducibility.

\section{Lewis's and Nelson's later philosophies of logic}

Although, in his later work, Lewis rejects the core ideas behind connexive logic, there are some similarities in their philosophies of logic in their later work.

Nelson writes little formal logic after the mid-1930s, but he does write papers on the philosophy of logic in that period. In those paper, he retains his Platonism. In a paper in 1949 he says:

I am convinced that no [...] analysis or criticism can be supported except by employing assumptions rooted in speculative philosophy. Thus in the purely abstract fields such as logic and mathematics we run into the problem of universals .... (Nelson 1949, 610)

In this passage Nelson is stating that intuitions about logical truth has to do with perceived or understood connections between abstract entities (universals). Nelson thinks that "acquaintance with instances of qualities or relations reveals that the essence of qualities and relations involves conformity with logical laws" (ibid. pp 612-613). The use of the word "acquaintance" would indicate to Nelson's readership that he has a position similar to Russell's theory of acquaintance with universals. At any rate, Nelson holds some version of platonism in his thesis and still maintains this view twenty years later.

Lewis did not change the formal logic that he accepted after 1930. His preferred logic remains S2 until the end of his life. But Lewis does change his philosophy of logic to look a lot like Nelson's. In a 1935 manuscript, Lewis says:

Let us begin with the interpretation of $p-3 q$, which is intended to be synonymous with $q$ is deducible from $p$. We conceive that all valid deduction takes place through the analysis of meanings or connotations. Observation of the truth or falsity of propositions 
may, in specific instances, show that one is not deducible from another - a false proposition is never deducible from a true one - but obviously such examination of truth-values, without analysis of propositions, can never demonstrate positively that $q$ is deducible from $p$. Socrates is a man implies Socrates is an animal because the connotation of man includes the connotation of animal. Connotations are (in the language of the older logic) universals; and any relation of connotations as such is a universal relation. If $q$ is deducible from $p$, this is because analysis of the meanings of the propositions $p$ and $q$ will reveal a universal relation of connotation or intension (Lewis and Langford 2014, 46) ${ }^{13}$

Lewis continues to develop this sort of semantic realism in his published work. A decade or so later in (Lewis 1946), he develops a more complex theory of abstract objects as the metaphysical basis of his theory of meaning. In (Lewis 1951), he treats statements again as expressing universals, that is as properties of worlds. Thus, in Lewis's later writings he adopts a metaphysical realist philosophy of logic similar to Nelson's.

\section{References}

Baylis, Charles A. 1931. "Implication and Subsumption." The Monist 41(3): 392-399. https://doi.org/10.5840/monist193141325

Bronstein, Daniel. 1936. "The Meaning of Implication." Mind 45(178): 157-180. https://doi.org/10.1093/mind/XLV.178.157

Duncan-Jones, Austin. 1935. "Is Strict Implication the Same as Entailment?" Analysis 2(5): 70-78. https://doi .org/10.2307/3326404

Goheen, John and Mothershead, John (eds.). 1970. Collected Papers of Clarence Irving Lewis. Stanford: Stanford University Press.

Hughes, George E. and Cresswell, Max J. 1968. An Introduction to Modal Logic. London: Methuen.

Ladd-Franklin, Christine. 1883. "On the Algebra of Logic." In Studies in Logic by Members of the John Hopkins University, edited by C. Peirce, pp. 17-71.

Lewis, Clarence Irving. 1910. The Place of Intuition in Knowledge. PhD Thesis, Harvard University, Cambridge, MA.

\footnotetext{
${ }^{13}$ Although Langford is listed as a co-author of this paper, both he and Lewis claimed that Langford had very little input into it.
} 
Lewis, Clarence Irving. 1918. Survey of Symbolic Logic. Berkeley: University of California Press.

Lewis, Clarence Irving. 1923a. "Facts, Systems and the Unity of the Worlds." The Journal of Philosophy 20(6): 141-151. Reprinted in (Gohen and Mothershead 1970), pp. 383-393. Page references are to the reprinted version.

Lewis, Clarence Irving. 1923b. "A Pragmatic Conception of the a Priori." The Journal of Philosophy 20(7): 169-177. Reprinted in (Gohen and Mothershead 1970), pp. 231-239. Page references are to the reprinted version.

Lewis, Clarence Irving. 1929. Mind and the World Order. Outline of a Theory of Knowledge. New York: Charles Scribner and Sons.

Lewis, Clarence Irving. 1930. "Logic and Pragmatism." In Contemporary American Philosophy, Volume II, edited by G. Adams and W. Montague, pp. 31-50. Reprinted in (Gohen and Mothershead 1970), pp. 3-19. Page references are to the reprinted version.

Lewis, Clarence Irving. 1946. Analysis of Knowledge and Valuation. LaSalle, IL: Open Court.

Lewis, Clarence Irving. 1951. "Notes on the Logic of Intension." In Structure, Method and Meaning: Essays in Honour of Henry M. Sheffer, edited by H. Kallen and S. Langer, pp. 25-34. Reprinted in (Gohen and Mothershead 1970), pp. 420-429. Page references are to the reprinted version.

Lewis, Clarence Irving and Langford, Cooper Harold. 1951. Symbolic Logic. New York: Dover. Originally published in 1932.

Lewis, Clarence Irving and Langford, Cooper Harold. 1959. Symbolic Logic. New York: Dover. Second edition.

Lewis, Clarence Irving and Langford, Cooper Harold. 2014. "A Note on Strict Implication (1935)." History and Philosophy of Logic 35(1): 44-49. https://doi .org/10.1080/01445340.2013.829282

McCall, Storrs. 2012. "A History of Connexivity." In Handbook of the History of Logic, Volume II, edited by D. Gabbay, F. Pelletier and J. Woods, pp. 415-449.

Nelson, Everett J. 1929. Towards an Intensional Logic of Propositions. PhD Thesis, Harvard University, Cambridge, MA.

Nelson, Everett J. 1930. "Intensional Relations." Mind 39(156): 440-453. https://doi .org/10.1093/mind/XXXIX.156.440

Nelson, Everett J. 1933. "On Three Logical Principles in Intension." The Monist 43(2): 268-284. https://doi.org/10.5840/monist19334327

Nelson, Everett J. 1936a. "A Note on Contradiction: A Protest." The Philosophical Review 45(5): 505-508. https://doi.org/10.2307/2180506

Nelson, Everett J. 1936b. "To the Editors of 'Mind'." Mind 45: 551. https://doi.org/10.1093/mind/XLV.180.551

Nelson, Everett J. 1949. "The Relation of Logic to Metaphysics." Philosophy and Phenomenological Research 9(3): 609-619. https://doi.org/10.2307/2104068

Organon F 26 (3) 2019: 405-426 
Priest, Graham. 1999. "Negation as Cancellation and Connexive Logic." Topoi 18(2): 141-148. https://doi.org/10.1023/A:1006294205280

Routley, Richard and Montgomery, Hugh. 1968. "On Systems containing Aristotle's Thesis." The Journal of Symbolic Logic 33(1): 82-96. https://doi.org/10.2307/2270055

Wansing, Heinrich. 2016. "Connexive Logic." In The Stanford Encyclopedia of Philosophy, edited by E. Zalta.

Wisdom, John. 1934. "Review of 'Symbolic Logic'." Mind 43: 99-109. https://doi.org/10.1093/mind/XLIII.169.99 